\title{
Intrinsic Gain in Self-Aligned Polysilicon Source-Gated Transistors
}

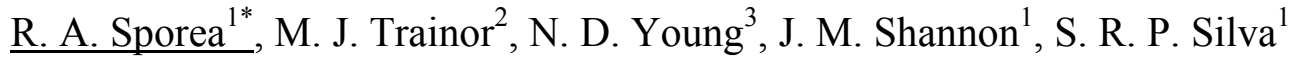 \\ ${ }^{1}$ Advanced Technology Institute, FEPS, University of Surrey, Guildford, Surrey, GU2 7XH, \\ $\underline{\text { United Kingdom }}$ \\ ${ }^{2}$ MiPlaza, Philips Research, High Tech Campus 4, 5656 AE Eindhoven, The Netherlands \\ ${ }^{3}$ Philips Research, 101 Cambridge Science Park, Milton Road, Cambridge CB4 0FY, \\ United Kingdom \\ *Email: r.sporea@surrey.ac.uk, Tel.+44 1483686083
}

\begin{abstract}
Thin-film, self-aligned source-gated transistors (SGTs) have been made in polysilicon. The very high output impedance of this type of transistor makes it suited to analog circuits. Intrinsic voltage gains of greater than one thousand have been measured at particular drain voltages. The drain voltage dependence of the gain is explained based on the device physics of the source-gated transistor and the fact that pinch-off occurs at both the source and the drain. The results obtained from these devices, which are far from optimal, suggest that, with proper design, the source-gated transistor is well suited to a wide range of analog applications.
\end{abstract}

Keywords: Source-gated transistor, polysilicon, Schottky barrier, intrinsic gain, voltage gain, TFT. 


\section{INTRODUCTION}

Thin-film polysilicon layers enable large area, inexpensive devices and circuits to be made for use in systems concerned with the man-machine interface [1,2] or cheap, disposable devices resulting from the economies of scale.

To date, the application of polysilicon devices is almost entirely restricted to digital circuits. In this paper we are concerned with analog circuits, and in particular the amplification factor or intrinsic gain of the thin-film transistor. It is difficult to obtain high intrinsic gain in conventional polysilicon FETs because the output characteristic is degraded by the so-called "kink effect" in which minority carriers generated in the high field region at the drain under saturation drift back toward the source and increase the drain current via the bipolar effect [3-5]. Therefore, output impedance is only large for small drain voltages. In a source-gated transistor, however, we have a reverse biased barrier at the source [6] that extracts any minority carriers. Furthermore, saturation is far stronger because it occurs at both the source and the drain and the output impedance can be very large.

It has been established that Source-Gated Transistors (SGTs) in polysilicon have the main characteristics that distinguish them from conventional thin-film field-effect transistors, namely a smaller saturation voltage and larger output impedance [7]. The SGTs were made by adapting a mask set used for making standard FETs using a technology based on excimer laser crystallisation of amorphous silicon. The bottom-gate structure had far from optimum performance in relation to the degradation of the transistor characteristics, due to poor passivation at the edge of the source and the large feedback capacitance formed by the large gate-drain overlap. 
In this paper we examine Source-Gated Transistors made using a similar but improved technology. In particular, the gate is self-aligned with the drain contact region. The intrinsic gain is measured on various structures and is analysed in relation to the device Physics that determines the output characteristic.

\section{TECHNOLOGY}

Self-aligned source-gated transistors were made on glass substrates. Figure 1 shows a microphotograph of a typical structure. A schematic cross-section of the structure is shown in Figure 2.

Following deposition and definition of a chromium gate metal, 200nm $\mathrm{SiN}_{\mathrm{x}}$ and $200 \mathrm{~nm} \mathrm{SiO} \mathrm{S}_{2}$ were deposited using PECVD, followed by $40 \mathrm{~nm}$ of a-Si:H. The amorphous silicon was dehydrogenated by baking at $450^{\circ} \mathrm{C}$ and various implants of $\mathrm{BF}_{2}$ or $\mathrm{P}$ were used to give a range of doping levels in the polysilicon.

The drain $\mathrm{n}^{+}$contacts were aligned with the gate using back exposure through the glass to define a positive resist. After chemical removal of the exposed resist regions, a high dose P implant was used to form drain contact regions aligned with the gate. The polysilicon and contact regions were formed using an excimer laser followed by definition and dry etching to form polysilicon islands. Source windows were etched in $120 \mathrm{~nm} \mathrm{SiO}_{2}$, followed by various $5 \mathrm{keV} \mathrm{BF}_{2}$ or $\mathrm{P}$ implants in order to modify the source barrier profile. The implants were annealed at $550^{\circ} \mathrm{C}$. Chromium and AlTi metal layers were deposited and defined to form a Schottky source and field plate structure shown in Figure 2. Finally, the structure was passivated using a $0.6 \mu \mathrm{m}$ nitride layer and contacts made to the source, drain and gate pads using $\mathrm{Cr} / \mathrm{AlTi} / \mathrm{Cr}$ metallisation via contact holes. 


\section{SOURCE-GATED TRANSISTOR CHARACTERISTICS}

The output and transfer characteristics were measured on devices similar to that shown in Fig. 1. Despite misalignment of the source window between the two drain contacts, the current between the source and either drain was the same, in agreement with the general property of an SGT that the current is independent of source-drain separation. (d in Fig. 2). Various devices with a range of source lengths (S), source-drain separations (d) and barrier changing implants have been measured.

Examples of device characteristics are shown in Fig. 3 and Fig. 4 for two devices with different polysilicon doping levels and different barrier modifying implants. Device (b) has a higher n-type polysilicon doping and therefore requires a larger negative voltage to turn it off compared with device (a).

It is seen in Fig. 3 that the characteristics exhibit the features of an SGT namely low saturation voltage and high output impedance. [6-8]: The change of saturation voltage with gate voltage was measured to be 0.04 , in good agreement with that predicted by the dielectric model of the SGT [8]. Furthermore the high output impedance is maintained right out to $\mathrm{V}_{\mathrm{D}}=20 \mathrm{~V}$ with no degradation due to the "kink effect".

While saturation in device (a) is sharp and well defined, device (b) shows a more rounded feature. This is most probably due to the high dose $\mathrm{BF}_{2}$ implant below the $\mathrm{Cr}$ source and the resulting doping profile. Since the annealing temperature for this implant was limited to $550^{\circ} \mathrm{C}$ to prevent glass compaction there will be a lot of residual damage centres and inactive boron making depletion more complicated.

Assuming barrier lowering is proportional to the electric field at the Schottky barrier interface [7], the transfer characteristic in Fig. 4 gives a barrier lowering constant of $\approx 3 \mathrm{~nm}$ for 
(a) and $\approx 2 \mathrm{~nm}$ for (b) [8]. While the value for (a) is similar to that measured in an ideal metalsilicon Schottky diode [9], the value for (b) is much lower. The latter is probably due to a thin oxide layer in the source window and to the poor quality of the polysilicon layer following the $\mathrm{BF}_{2}$ implant.

It has been shown previously, using computer modelling that the current crossing the source barrier is concentrated at the drain end of the source [10]. This feature is shown experimentally in Figure 5. Here we see that the drain current is insensitive to source length provided it is greater than $\approx 2 \mu \mathrm{m}$. Therefore, to maximise frequency response, the source length should be small, since a long source simply increases the source capacitance without increasing the current. For device (a) shown in Figure 3, which has a $2 \mu \mathrm{m}$ source length, the calculated $\mathrm{f}_{\mathrm{T}}[11]$ increases to approximately $80 \mathrm{MHz}$ at the highest gate voltages.

\section{INTRINSIC GAIN IN AN SGT WITH A SCHOTTKY SOURCE}

The intrinsic voltage gain is given by the ratio of the transconductance $g_{m}$ and the output conductance $g_{d}$. An analysis of the SGT with a Schottky barrier source shows that the transconductance is proportional to the average source current density $\mathrm{J}_{\mathrm{S}}$ and the capacitance per unit area of the gate insulator [11]. Therefore $g_{m}$ will increase with decreasing source barrier height and gate insulator thickness.

In an FET the output conductance in saturation is determined by channel length shortening with increasing $\mathrm{V}_{\mathrm{D}}$, combined with the effect of carrier generation in the high-field pinch-off region at the drain (the so-called "kink effect"). In the SGT we expect both these physical mechanisms to play a role but the saturation is more complicated because as $V_{D}$ increases, pinch-off and current saturation first occurs under the source, as shown 
schematically in Figure 6(a) and then, at higher $V_{D}$, the device also pinches off at the drain end of the parasitic FET (Figure 6(b)).

The change of drain current with drain voltage is shown schematically in Figure 7 for two gate voltages. As $\mathrm{V}_{\mathrm{D}}$ increases from $0 \mathrm{~V}$, for a given gate voltage $\mathrm{V}_{\mathrm{G} 1}$, the depletion layer of the reverse biased source barrier becomes thicker and the electric field under the source increases until the carriers induced by the gate are depleted. This is region (1) in Figure 7. The current at $\mathrm{V}_{\mathrm{SAT} 1}$ saturates because further increase in $\mathrm{V}_{\mathrm{D}}$ does not affect the electric field under the source barrier. A further increase in $V_{D}$ causes the depletion envelope to spread sideways towards the drain. In this region, (2) in Figure 7, the output conductance depends on the quality of the passivation at the edge of the source (point A in Figure 6) and its ability to minimise any rise in the normal component of the electric field under the barrier as the drain field increases. In region (2) there will be a small potential drop across the parasitic FET so the change of potential of its floating source (point B in Figure 6) will be similar to the change of $\mathrm{V}_{\mathrm{D}}$. However, as the floating source potential becomes more positive, the FET begins to turn off, its conductance starts to decrease and it drops more potential. Therefore, in region (2) of Figure 7 we expect a decrease in the conductance as $V_{D}$ increases because the change in electric field with $V_{D}$ is more uniformly spread between source and drain. Finally, when $V_{D}$ $=\mathrm{V}_{\mathrm{G}}-\mathrm{V}_{\mathrm{T}}$ the parasitic FET pinches off close to the drain contact and the current in the parasitic FET saturates ( $\mathrm{V}_{\mathrm{SAT} 2}$ in Figure 7). In this region we expect the overall conductance of the SGT to be affected by the conductance of the FET in saturation, which is dependent on channel shortening effects. Therefore, around $\mathrm{V}_{\mathrm{SAT} 2}$ the conductance should be influenced by the source-drain separation and the length of the channel. With both source and drain ends pinched off, a very large output impedance is expected, (region 3) which is indeed a feature of the source-gated transistor. Above $V_{D}=V_{\text {SAT2 }}$ further increases in $V_{D}$ will be dropped across 
the pinch-off region at the drain and the field will increase strongly causing carrier generation via hot carriers and a rise in the output conductance (region (4) in Figure 7).

Also shown in Figure 7 is a schematic of the $I_{D}$ vs. $V_{D}$ curve for a higher $V_{G}$. $I_{D}$ is higher so we expect a larger $g_{m}$. The change in $V_{\mathrm{SAT} 1}$ is small because $\mathrm{dV}_{\mathrm{SAT1}} / \mathrm{dV}_{\mathrm{G}}$ is small [7] but there is a shift in $\mathrm{V}_{\mathrm{SAT} 2}$ because of the change in $\mathrm{V}_{\mathrm{G}}-\mathrm{V}_{\mathrm{T}}$, and $\Delta \mathrm{V}_{\mathrm{SAT} 2}=\mathrm{V}_{\mathrm{G} 2}-\mathrm{V}_{\mathrm{G} 1}$. Furthermore, a larger $\mathrm{V}_{\mathrm{G}}$ will increase the electric field, particularly near the drain and an increase in carrier generation at $\mathrm{V}_{\mathrm{D}}>\mathrm{V}_{\mathrm{SAT} 2}$ is expected. It should be noted, however, that whilst in an FET minority carrier current tends to be amplified because the source junction becomes forward biased and there is a bipolar effect (the kink effect), in the SGT the source is reverse biased, minority carriers are extracted and no amplification occurs.

\section{INTRINSIC GAIN MEASUREMENTS}

Intrinsic voltage gain $\left(g_{m} / g_{d}\right)$ measurements were made on transistors having a range of source lengths and source-drain separations. There were also variations in the characteristic of the Schottky source barrier due to implantation of dopants below the source barrier. In general, the transconductance of the devices was poor in comparison to an equivalent FET because firstly the barrier lowering constant was low, presumably due to a thin oxide layer in the source window, and secondly the insulator was thick $\left(200 \mathrm{~nm} \mathrm{SiO} \mathrm{Si}_{2}+200 \mathrm{~nm} \mathrm{SiN}\right.$ ) compared to that in a typical polysilicon FET. This means that $\mathrm{J}_{\mathrm{S}}$ and $\mathrm{C}_{\mathrm{i}}$ were much smaller than optimum. The output conductance, however, was very low for certain values of $\mathrm{V}_{\mathrm{D}}$ and strongly $\mathrm{V}_{\mathrm{D}}$ dependent. This led to voltage gains higher than a thousand in some devices.

An example of the drain voltage dependence of the intrinsic gain in these polysilicon SGT's is shown in Figure 8 for two different gate voltages. The output characteristics were 
similar to those shown in Fig. 3(b). The source-drain separations in 8(a) and 8(b) were 4 and 10 microns, respectively. To modify the barrier, a BF2 implant was made in the source window. This implant should also aid passivation, since the polysilicon region around the edge of the window will be less n-type. We see from Figure 8 that for a given $V_{G}$ there are large variations in the gain with drain voltage. Since $g_{m}$ does not change very much, these variations are due to changes in $g_{d}$.

With reference to Figure 8 (a), at $V_{G}=-36 \mathrm{~V}$ the gain increases rapidly as $V_{D}$ increases to $\mathrm{V}_{\mathrm{SAT} 1},((1)$ Fig. 7$)$ which is approximately $1.5 \mathrm{~V}$ It then increases further as the device saturates at $\mathrm{V}_{\mathrm{SAT} 2},\left((2)\right.$ Fig. 7) which since $\mathrm{V}_{\mathrm{T}} \approx-40 \mathrm{~V}$, should be around $4 \mathrm{~V}$. The highest gain occurs when $\mathrm{V}_{\mathrm{D}}>\mathrm{V}_{\text {SAT2 }}$, ((3) Fig. 7) but as $\mathrm{V}_{\mathrm{D}}$ becomes much larger than $\mathrm{V}_{\mathrm{SAT} 2}$, the gain falls off, presumably due to an increase in $g_{d}$, as suggested above ((4) Fig. 7 ). Increasing $V_{G}$ to $30 \mathrm{~V}$ shows a rise in gain at low voltages, consistent with an increase in $\mathrm{I}_{\mathrm{D}}$ and $\mathrm{g}_{\mathrm{m}}$. However, there is a shift in the position of the maximum gain, which is consistent with a shift of $6 \mathrm{~V}$ in $\mathrm{V}_{\mathrm{SAT2}}$. Interestingly, the increase in gain around $\mathrm{V}_{\mathrm{SAT} 1}$ due to a larger $\mathrm{g}_{\mathrm{m}}$ is very similar to that around $\mathrm{V}_{\mathrm{SAT} 2}(\approx 4)$. We see, therefore, that these gain curves are consistent with the general description of what is expected from the output characteristics.

Measurements on an identical structure but with a larger source-drain separation are shown in Figure $8(\mathrm{~b})$. The gains at low drain voltages are similar to those in Figure 8(a) but at higher $\mathrm{V}_{\mathrm{D}}$ maximum gains are much higher. For $\mathrm{V}_{\mathrm{G}}=-36 \mathrm{~V}$, for example, measurements in Fig 8 (a) show a maximum gain of $\approx 250$ for $\mathrm{V}_{\mathrm{D}} \approx 8 \mathrm{~V}$. In Figure 8 (b) the gain continues to rise to a value of $\approx 500$ at $\mathrm{V}_{\mathrm{D}} \approx 10 \mathrm{~V}$. We attribute this to a reduction of channel-shortening effects and $\mathrm{g}_{\mathrm{d}}$ in the device with a larger source-drain separation. At $\mathrm{V}_{\mathrm{G}}=-30 \mathrm{~V}$, maximum gains can be extremely large, as output conductance tends to zero. In fact, we have measured negative resistance in some devices, due to the interaction between the two high-impedance regions at 
the source and drain ends of the device. Above $V_{D}=15 \mathrm{~V}$, these measurements become very noisy and unreliable. However, if we scale the increase in gain with that measured around $\mathrm{V}_{\mathrm{SAT} 1}$, the maximum gain is $\approx 5000$. It remains to be seen if these very high gains are practically useful.

A device with very different gain characteristics is shown in Figure 9. This device had a phosphorus implant in the source window which made the polysilicon more n-type. The edge passivation was therefore likely to be worse than in the previous sample. This device, which had a current ten times higher than those shown in Figure 8, did not show large variations in gain with VG. We believe that this is due to the poor passivation and to the formation of a high field region at the edge of the source window (region A in Figure 6). Such a region could provide a situation in which the conductance is proportional to the current either due to carrier generation or due to the effect of the lateral electric field on the low peripheral barrier. Such an effect, of course, would tend to negate any increase in gain arising from an increase in current and $\mathrm{g}_{\mathrm{m}}$. Despite the degradation of $\mathrm{g}_{\mathrm{d}}$, however, gains over 500 are observed.

\section{CONCLUSION}

Self-aligned Source-Gated Transistors have been fabricated in polysilicon. Using $\mathrm{Cr}$ Schottky source barriers, the characteristic features of Source-Gated Transistors, namely high output impedance and low saturation voltage, were observed with an absence of the deleterious "kink effect" found in polysilicon FETs. The effective barrier lowering constant was similar to that expected in the high current devices, but many samples had low values, presumably due to the presence of a thin oxide in the source window and residual disorder 
following the barrier modification implant. The calculated $f_{T}$ of the highest current devices was well above $50 \mathrm{MHz}$.

The drain voltage dependence of the intrinsic voltage gain $\left(\mathrm{g}_{\mathrm{m}} / \mathrm{g}_{\mathrm{d}}\right)$ was complicated by the fact that the device first pinches off at the source and then at the drain, as drain voltage was increased. This led to a double peak in the gain characteristic with the highest gain achieved when both source and drain were pinched off. Intrinsic voltage gain in excess of a thousand was obtained in some cases. It is shown that the variations of gain with drain voltage in a well passivated structure agree well with our understanding of how the device works. The high gains were obtained by engineering very high output impedances and are well suited to analog applications. These devices were far from optimum, however, particularly in relation to $g_{m}$ and their speed of operation. 


\section{REFERENCES}

[1] T. Nishibe and T. Nakamura, "Realization of new-concept "Input Display" by p-Si SOG technology”, Proc AMLCD’04, pp 85-88, 2004.

[2] H. Asada, K. Sera, S. Kaneko, "Development of System-on-Glass (SOG) Display Technology”, NEC Technical Journal, Vol.1, No.3, pp 85, 2006.

[3] J. R. Ayres, S. D. Brotherton, D. J. McCulloch and M. J. Trainor, “Analysis of drain field and hot carrier stability of poly-Si thin film transistors", Jpn. J. Appl. Phys., vol. 37, no. 4A, pp 1801-1808, 1998.

[4] M. Valdinoci, L. Colalongo, G. Baccarani, G. Fortunato, A. Pecora, and I. Policicchio, "Floating body effects in polysilicon thin-film transistors", IEEE Trans ED vol. 44 no. 12 , pp. 2234-2241, 1997.

[5] A. Valletta, P. Gaucci, L. Mariucci, G. Fortunato and S. D. Brotherton, "Kink effect in short channel polycrystalline silicon thin film transistors", Appl Phys Lett, vol. 85, no. 15, pp. 3113-3115, 2004.

[6] F. Balon and J. M. Shannon, "Analysis of Schottky barrier source-gated transistors in aSi:H”, Solid-State Electronics, 50, pp. 378-383, 2006.

[7] J. M. Shannon and E. G. Gerstner, "Source-gated thin-film transistors", IEEE Electron Dev. Lett., 24, no. 6, pp. 405-407, 2003.

[8] J. M. Shannon and E. G. Gerstner, "Source-gated transistors in hydrogenated amorphous silicon", Solid-State Electronics, Vol. 48, No. 6, pp. 1155-1161, 2004.

[9] J. M. Shannon, “Thermionic-Field Emission through Silicon Schottky Barriers at Room Temperature", Solid-State Electronics, Vol. 20, pp. 869-872, 1977. 
[10] J. M. Shannon and F. Balon, "Source-gated thin-film transistors", Solid State Electronics, Vol. 52, Issue 3, pp. 449 - 454, 2008.

[11] J.M. Shannon and F. Balon, "Frequency Response of Source-Gated Transistors", IEEE Trans. Electron Devices, Vol. 56, Issue 10, pp. 2354-2356, 2009.

[12] S. M. Sze, "Physics of semiconductor devices", Second Edition, John Wiley \& Sons, 1981. 


\section{FIGURE CAPTIONS}

Figure 1. Microphotograph of a self-aligned SGT in polysilicon on glass. Despite the misalignment of the source contact window and source metallisation, the current to each of the drains is the same. $\mathrm{W}=50 \mu \mathrm{m}, \mathrm{S}=2 \mu \mathrm{m}, \mathrm{d}=4 \mu \mathrm{m}$.

Figure 2. Schematic cross-section of a self-aligned SGT.

Figure 3. Output characteristic of typical polysilicon SGTs showing low saturation voltage and high impedance in saturation. The n-type doping in the polysilicon layer was $1.5 \cdot 10^{12}$ and $2.5 \cdot 10^{12} / \mathrm{cm}^{2}$ and the barrier modification implant was $1 \cdot 10^{13}$ and $7.5 \cdot 10^{13} / \mathrm{cm}^{2}, 5 \mathrm{keV} \mathrm{BF}_{2}$ for (a) and (b) respectively. $\mathrm{W}=50 \mu \mathrm{m}, \mathrm{S}=2 \mu \mathrm{m}, \mathrm{d}=4 \mu \mathrm{m}$.

Figure 4. Transfer characteristics of the transistors shown in Figure 3.

Figure 5. Dependence of drain current on source length. Most of the current flows at the edge of the source opposite the drain. $\mathrm{W}=50 \mu \mathrm{m}, \mathrm{d}=6 \mu \mathrm{m}$.

Figure 6. Schematic showing the two pinch-off states of the SGT. In (a) the semiconductor is pinched-off at the source. In (b) when $\mathrm{V}_{\mathrm{D}}=\mathrm{V}_{\mathrm{G}}-\mathrm{V}_{\mathrm{T}}$ the semiconductor pinches off at both the source and the drain. $A$ is the corner of the source most sensitive to drain field. $B$ is the position of the floating source of the parasitic FET.

Figure 7. Schematic showing how the drain current changes with drain voltage for two values of $\mathrm{V}_{\mathrm{G}}$ based on our understanding of how the SGT works.

Figure 8. Intrinsic gain measurements on two identical devices apart from the source drain separation. $\mathrm{W}=50 \mu \mathrm{m}, \mathrm{S}=2 \mu \mathrm{m}, \mathrm{V}_{\mathrm{T}} \approx-40 \mathrm{~V}$.

Figure 9. Intrinsic gain measurements on a high-current device with poor edge passivation. 


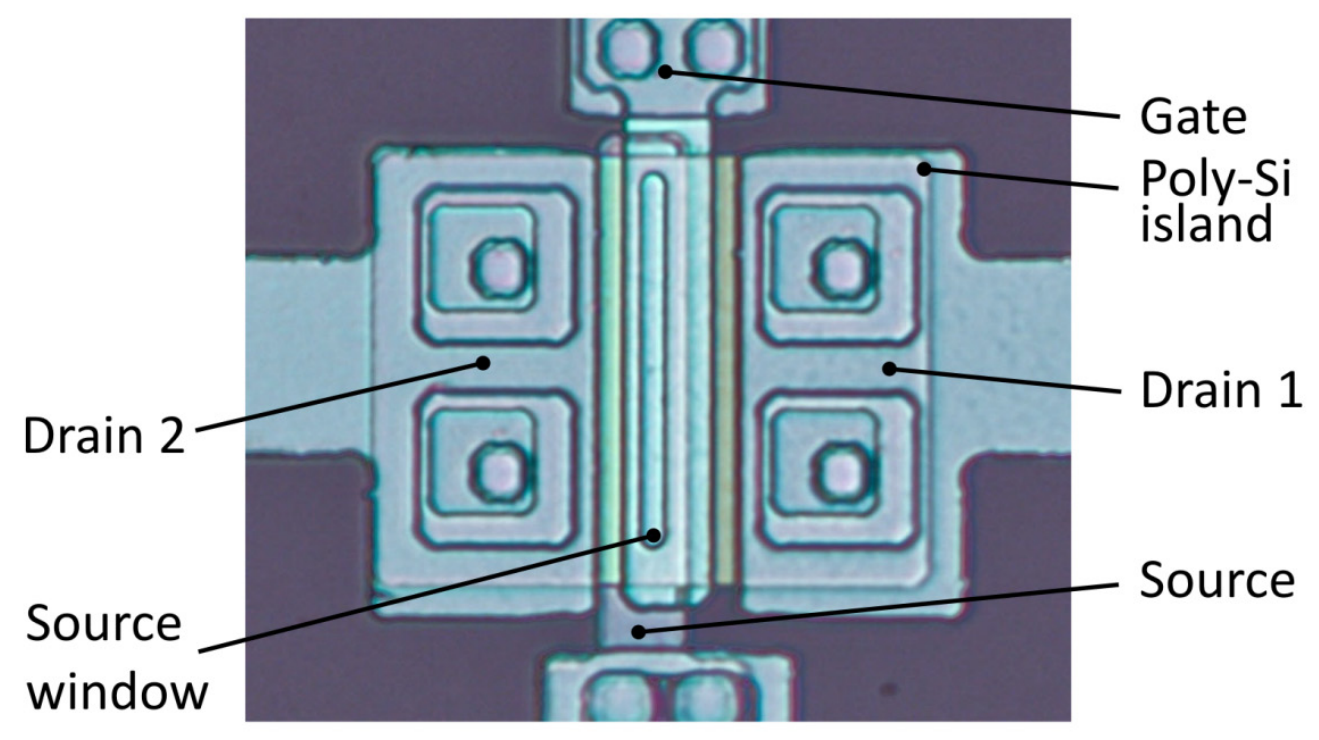

Figure 1 


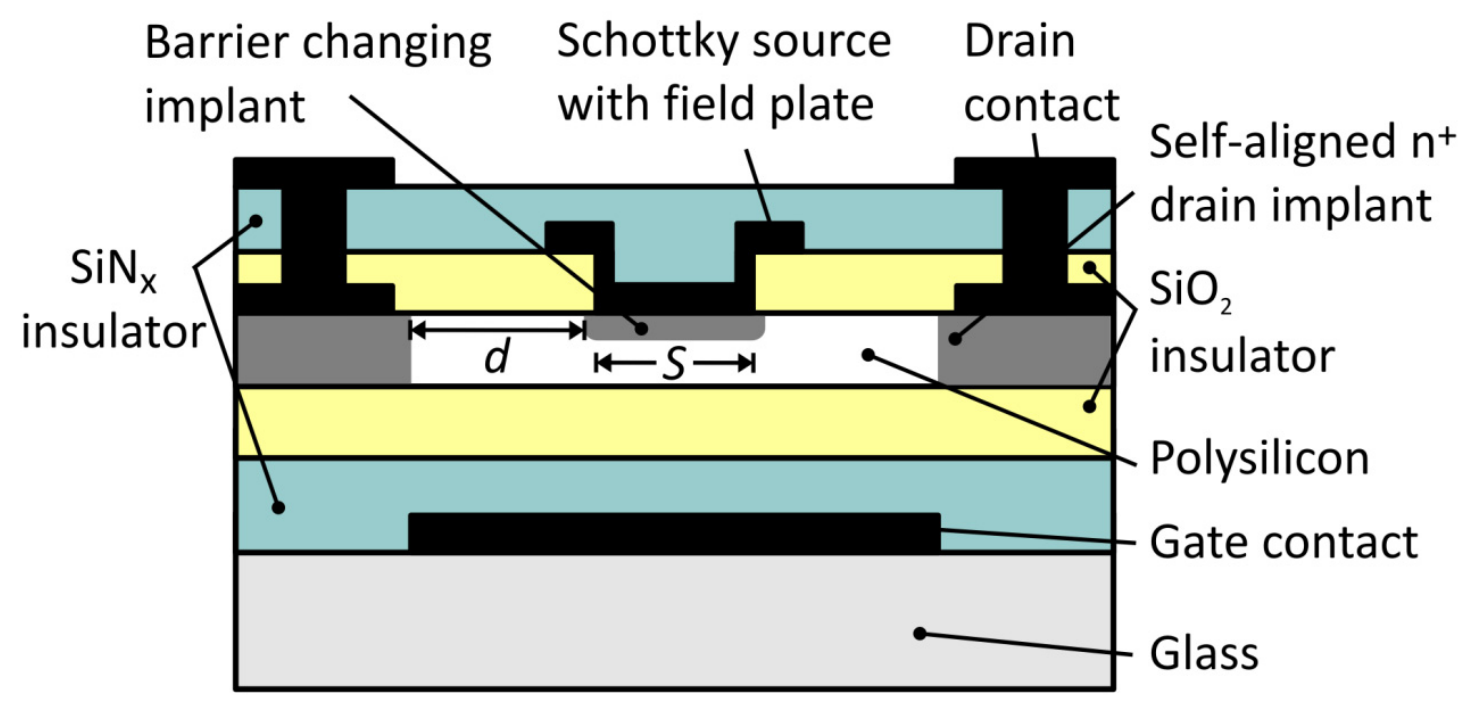

Figure 2 

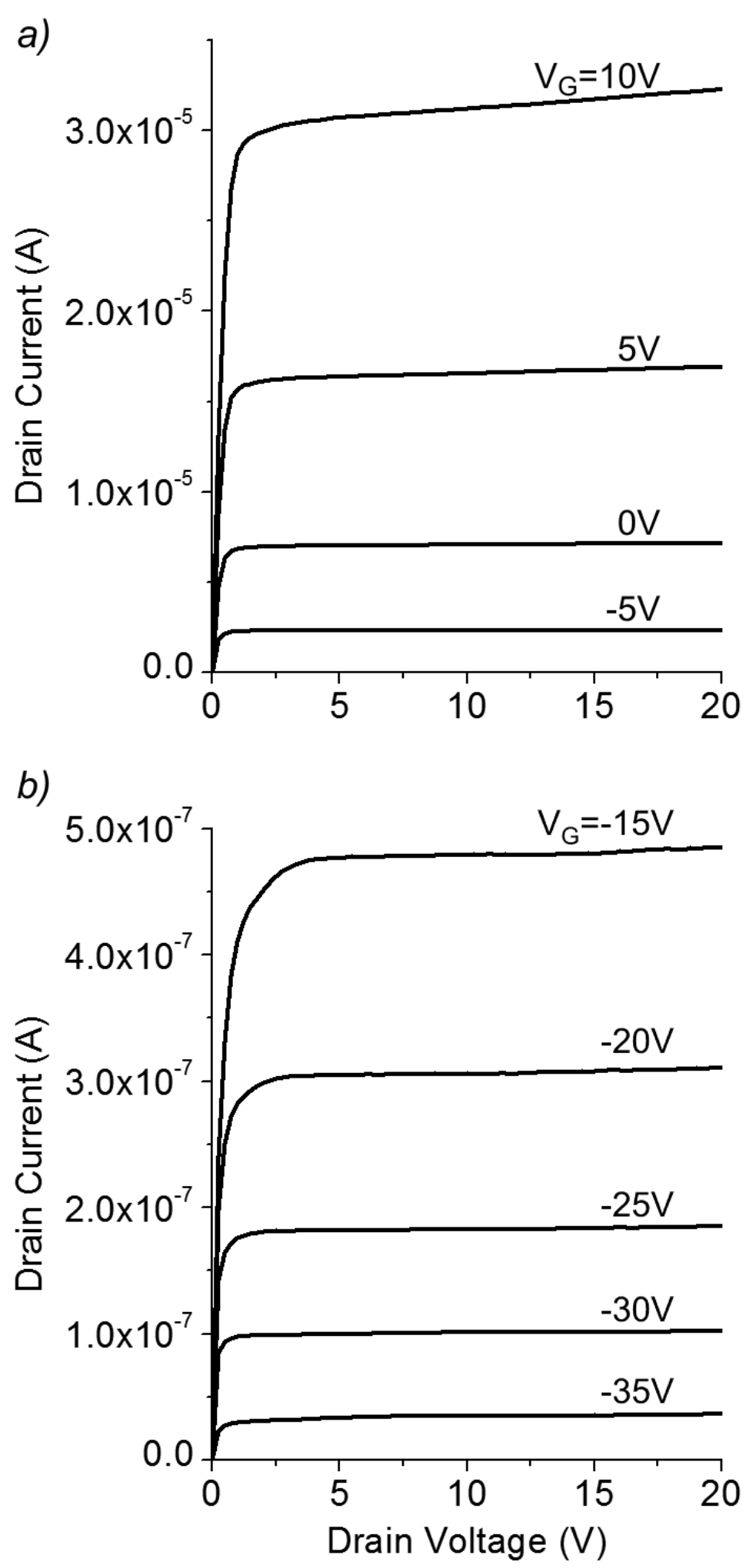

Figure 3 


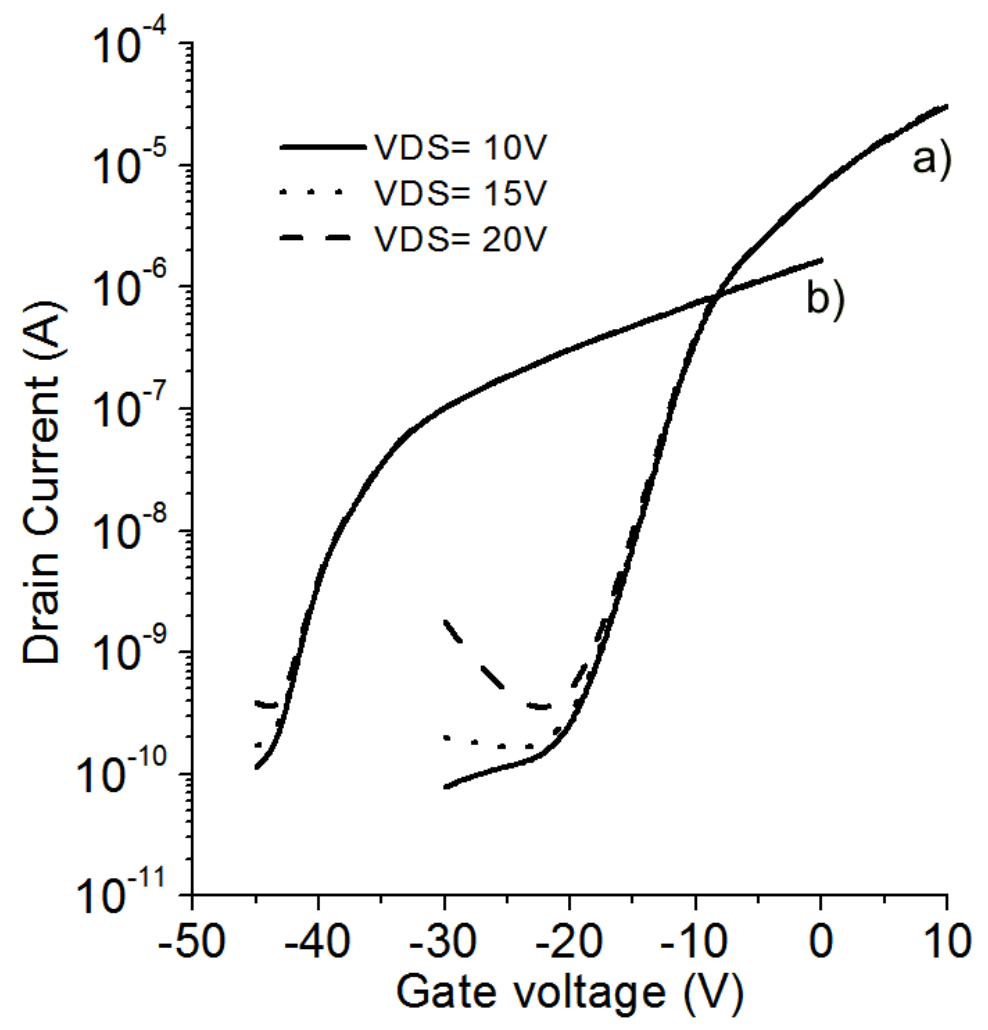

Figure 4 


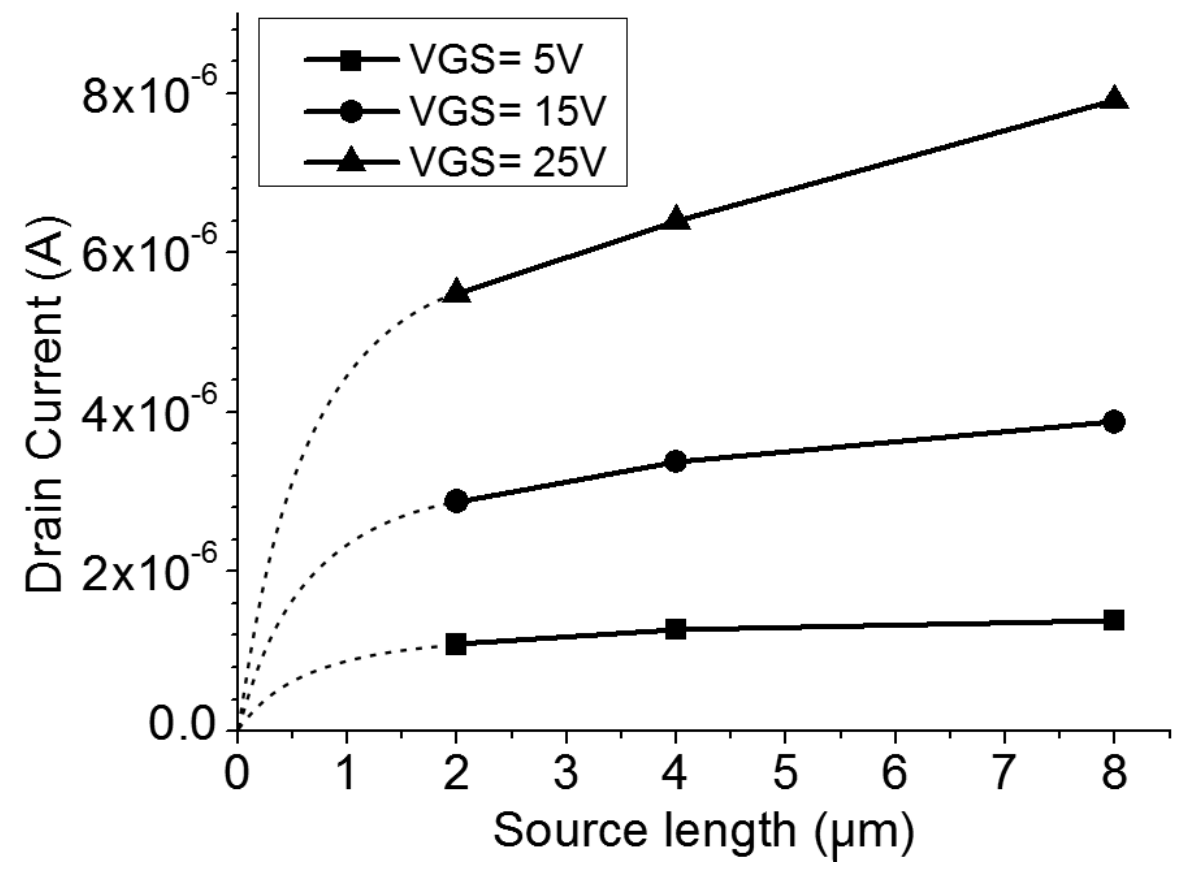

Figure 5 


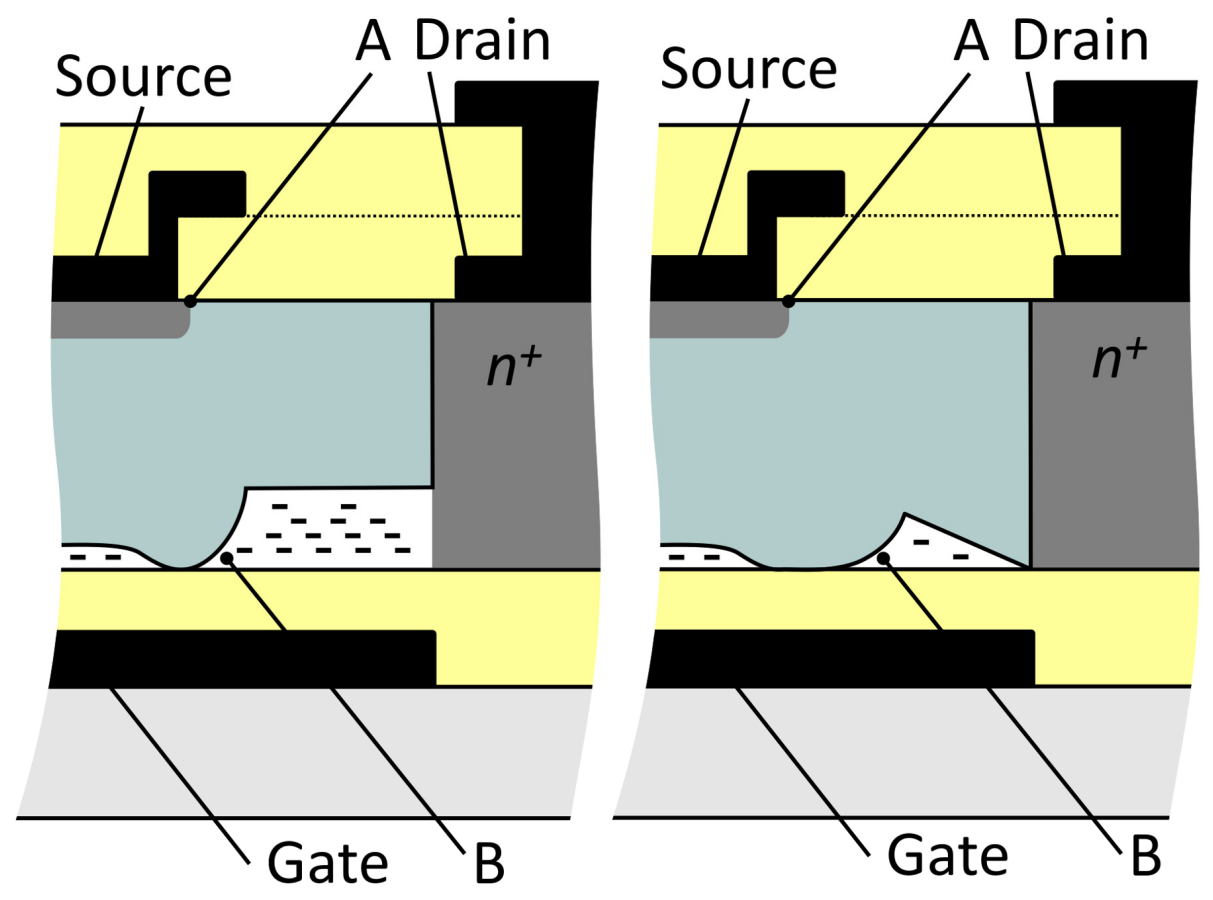

a)

b)

Figure 6 


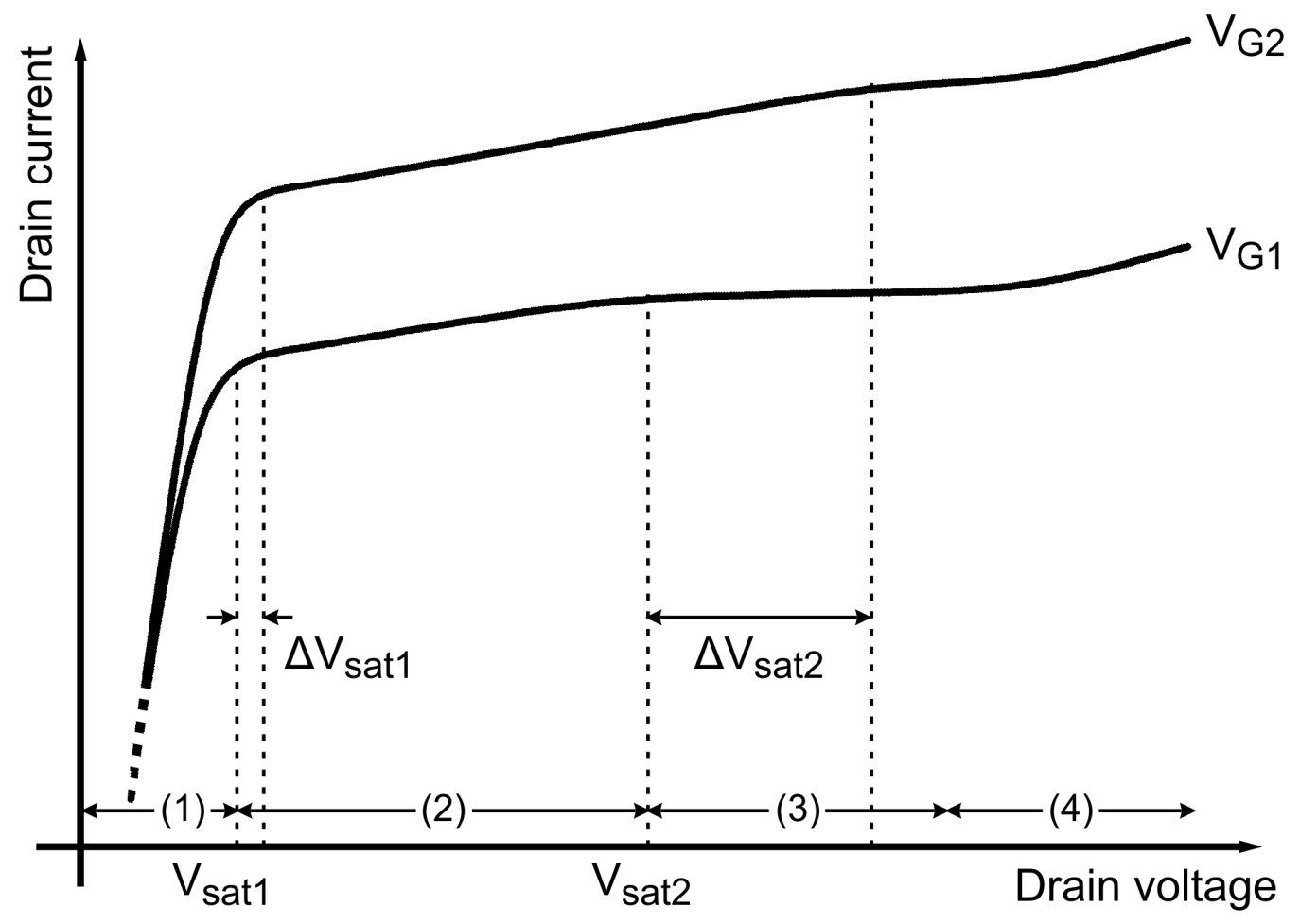

Figure 7 


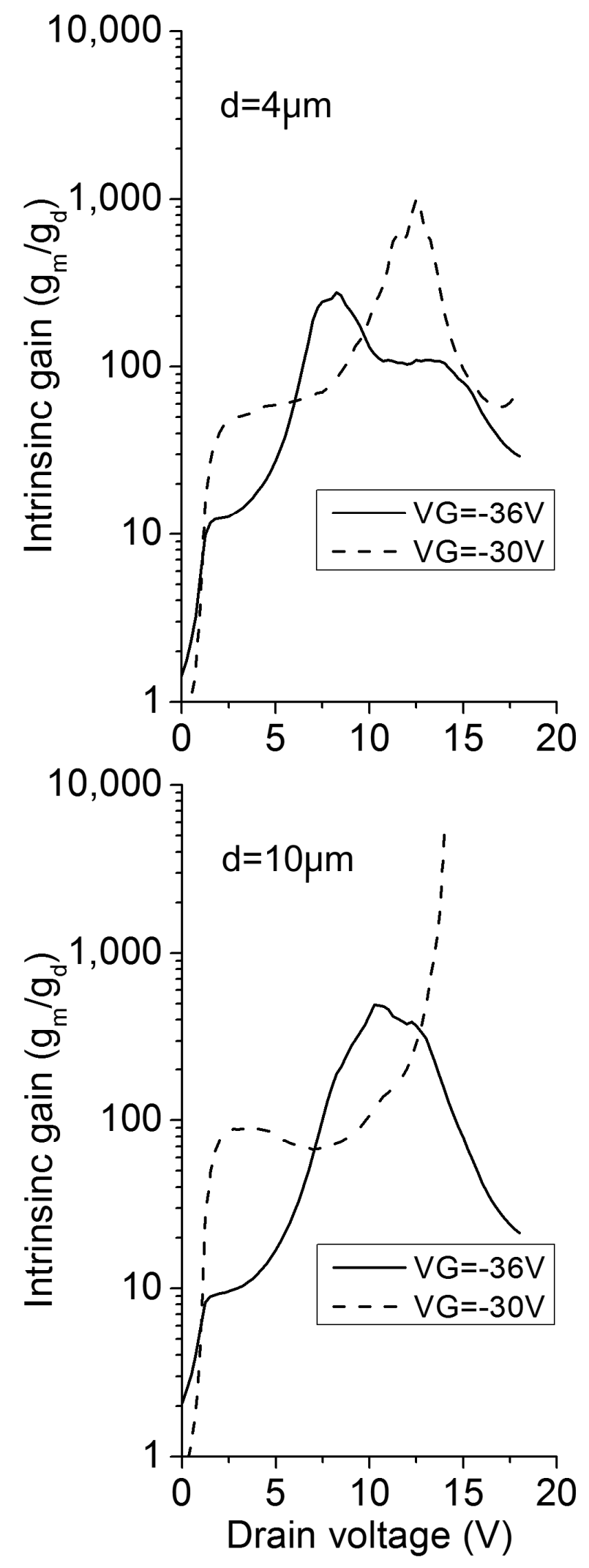

Figure 8 


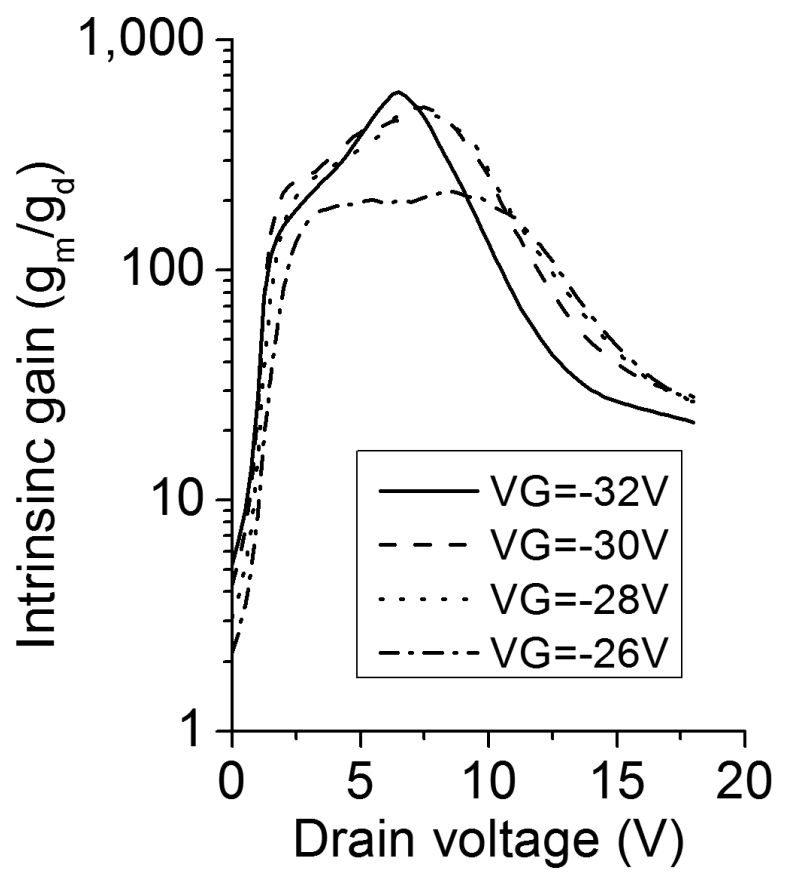

Figure 9 
Radu A. Sporea (M'05) received his Engineer's Diploma in 2006 from the Faculty of Electronic Engineering, Information Technology and Telecommunications, 'Politehnica' University of Bucharest. He has worked as a Junior Design Engineer for Catalyst Semiconductor Romania, now part of On Semiconductor, on low power analog circuits using the floating gate technology. He is currently holding an EPSRC-funded PhD studentship at the Advanced Technology Institute, University of Surrey, in Guildford, UK. His interests include low power IC design and novel devices in silicon and organic materials and their application to large scale electronics. Mr. Sporea is a student member of IEEE Solid State Circuits Society, IEEE Communications Society, Society of Information Display and of the IET.

Mike

Nigel

John M. Shannon received the D.Sc. degree from Brunel University, London, U.K. in 1982. From 1985 to 1994, he was the Head of the Display and Large Area Electronics Group at Philips Research Laboratories Redhill before sharing his time with the University of Surrey, Guildford, U.K., where he is a Professional Research Fellow with the Faculty of Electronics and Physical Sciences. He has numerous paper and patents in the field of semiconductor devices. 
Dr. Shannon was elected a Fellow of the Royal Academy of Engineering London in 2001. He is presently Professor of Semiconductor Devices in the Advanced Technology Institute at the University of Surrey.

Ravi 